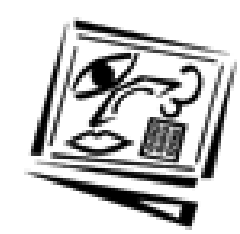

Australian Journal of
Educational Technology

\title{
Interactive multimedia learning in physics
}

\author{
Susan J. Bennett and Michael J. Brennan \\ Australian National University
}

The potential importance of interactive multimedia in the tertiary environment and in physics education is well recognised world wide, with development projects being funded by universities and government bodies. To date, however, there have been few opportunities to critically assess the importance, acceptability and suitability of these new techniques to physics education. Our study evaluated an interactive tutorial, developed at the Australian National University, covering the photoelectric effect and related material targeted at first and second year students. Pre- and post-test questionnaires using scaled and open answers, observations by teaching staff and informal discussions, were conducted with 35 students in the Department of Physics and Theoretical Physics. Results from an independent survey of the course were also obtained. Our principal conclusion is that while interactive multimedia shows a high degree of acceptability with our study group, any practical application must have a meaningful assessable component and be fully integrated into the lecture series, as opposed to being offered as a study resource alone.

The philosophies and practicalities of multimedia in education have been reviewed at length; see for example Latchem (1993) and Laurillard (1993). Rather than discuss these fully, only the issues relevant to this particular study will be identified here.

There are many perceived advantages of interactive multimedia over traditional teaching methods. One of these is as a cost effective alternative to individual or group, tuition, where such a tool allows the provision of immediate and consistent feedback otherwise unavailable due to the limited availability of staff, or where the course is run as part of a distance education program (Ladiges et. al., 1994). It also has the potential to extend more advanced students and to assist those who require more attention through the opportunity to include various levels of complexity in one package.

The multimedia tutorial allows students to work at their own pace and supports connections and comparisons that a traditional text does not. An environment in which the student is "empowered to control their own learning" is said to foster "deep learning" which is self-motivated and selfdirected (Latchem, 1993). Studies also show that the retention rate of 
students learning through multimedia is significantly greater than more traditional forms and their motivation and self esteem are also higher (Clarke and Swearingen, 1994).

Multimedia can, through its power to animate, communicate dynamic information more accurately than a diagram and can help students visualise phenomena that cannot be seen, for example chemical reactions (Whitnell 1994). Care must be taken, however, to avoid an overly sophisticated treatment which will only serve to make learning more difficult (Beattie, 1994). Laurillard (1993) suggests a combination of simulation and tutorial as one of the only ways to adequately replace the one-to-one interaction of student and teacher.

Multimedia simulations have also been developed to replace prohibitively expensive practical work while still bringing together concepts covered in lectures, developing problem solving skills and allowing students to experience experimental design (Arundel et. al., 1994).

In this paper, we present a study of the responses of a group of physics and engineering students in the Department of Physics and Theoretical Physics at the Australian National University in Canberra to a multimedia tutorial on blackbody radiation and the photoelectric effect. In the following sections we describe the teaching context, design, development and implementation process, the evaluation methods and results and draw some general conclusions from the work.

\section{Teaching context}

An understanding of quantum concepts is fundamental to most courses in both physics and engineering. While students may have shown they can solve problems at an introductory level, they often arrive at second year with an incomplete appreciation for even the simplest concepts. Meaningful practical work is difficult, expensive and often requires a degree of skill which undergraduate students may not possess. This has often resulted in the adoption of a didactic approach reliant on static forms of communication.

In past years the initial quantum physics component of the second year general physics course in the Department of Physics and Theoretical Physics at the ANU was presented in 6 lectures and coupled with a study of special relativity and an introduction to nuclear physics, a total of 18 lectures over 6 weeks. Most students had some previous exposure to the concepts covered in both the quantum physics and special relativity components. Exam results indicated that those who had previously studied the material had trouble with many of the concepts (this observation was supported by the pre-test questionnaires for our sample group). Thus, the need to quickly bring these students up to speed was identified. 
The proximity of the Research School of Physical Sciences and Engineering in the Institute of Advanced Studies to the Department of Physics in The Faculties, where the undergraduate teaching is conducted, allows for some of the teaching load to be undertaken by members of the research school, with obvious benefits to the students of exposure to a wide range of lecturers. The location of the research school on the far side of campus means that students do not consult lecturers as often as they do those within the teaching department, thus there was a necessity to provide extra material for sufficiently motivated students to study.

The main objective of the teaching team was to improve the general understanding of quantum physics while providing some context for the students. In addition, we hoped to increase the opportunities for student engagement and independence. Multimedia was therefore trialed as a method of addressing the practical problems of a short course for which students need to build upon prior knowledge and the relative inaccessibility of the lecturer. We also hoped that it would improve student attitude and motivation for tutorials and assignments.

Those of us who have demonstrated in and/or learned in practical sessions in physics laboratories are familiar with the equipment failures and the distraction of obtaining results. Multimedia programs represent an opportunity for the students to learn about the physics involved, while momentarily removed from the inevitable complications of obtaining the often less than spectacular results on which analysis must be based. Multimedia does not help students develop the practical laboratory skills that are the basis of scientific research and we recognise, along with the vast majority of multimedia proponents, that there is no substitute for hands-on experience.

The implementation of the interactive tutorial, a first for the Department of Physics and Theoretical Physics, was accompanied by a general change in teaching strategy. Lectures were presented using Microsoft Powerpoint with the notes made available on the departmental server. Most students brought these notes to class and annotated them. This represented an integration of information technology into the learning environment which was designed to utilise a generally high degree of familiarity with personal computers within the student group and to better cater for a variety of learning styles, while not harshly disadvantaging the few students for whom the personal computer environment was novel.

The inclusion of multimedia in the course was also designed to have an impact on traditional teaching methods. The support of the notes and tutorial material would leave students free to concentrate on the main concepts, rather than being concerned with taking verbatim notes, or getting their experiment to work. It would also allow the lecturer to act as a facilitator for learning and would give more opportunity to promote discussion. 
The project was also the completion of a Masters project in scientific communication for one of the authors (Bennett, 1995). The development team was advised by engineering and physics lecturers, students (undergraduate and postgraduate), educational designers and postgraduate students in communication.

The most profound disadvantage we envisaged was the significant effort involved in the development of the software. We were also concerned that student should not develop feelings of detachment from the teacher or lack interaction with other students.

\section{Design issues}

The prototype tutorial, "Introduction to Quantum Physics - Photons" explores blackbody radiation and the photoelectric effect as a focus for a discussion of basic quantum physics. The material is sufficiently general to allow it to be included in any introductory physics or engineering course or as revision material for later year students.

The organisational structure is that of a scientific paper or laboratory write-up with aims, background theory, experimental method, interpretation of results and conclusions. This imposed structure reinforces professional practice and philosophy while reflecting the model commonly used for presenting a scientific argument.

The navigational structure is largely linear with diversions branching off from the main topic. The tutorial features an extensive contents page which allows students to move easily to any of the main sections, offering some scope for exploration and choice. This kind of structure is widely used for educational multimedia and has proved one of the most effective (Stocklmayer, 1995). Navigation tools are limited to forward and backward buttons. A quit button always appears on screen to allow an exit at any time. The branched nature of the structure allows the inclusion of scientific and historical background information and mathematical derivations to provide detailed explanations and context for those who are interested.

The interface is deliberately simple with large amounts of white space to make the text easier to read. The presentation is very straight forward, using only simple graphics, few transitions, no humour and limited sound. These have been deliberately avoided as they become very tedious when frequently reviewing material. A further advantage is the opportunity to run the tutorial from a server for multiple users with little or no reduction in performance. The help provided is very limited as it was assumed that this tutorial would be used in conjunction with some level of supervision or instruction.

Animations, both interactive and non-interactive, have been designed to enhance the students understanding of the material while building upon 
the types of representation commonly found in texts. A variety of interactions, such as type-in answers and quiz questions, have been incorporated into the body of the tutorial to emphasise main points and to maintain interest.

Another section leads the students through an analysis of data from the original photoelectric effect experiments (Halliday and Resnick, 1988). They are required to find the line of best fit and then use the information to calculate various quantities. The procedure is very similar to data analysis that students at this level are expected to perform in laboratory classes.

A quiz at the end of the module is designed for the students to test their understanding of the material. In this tutorial, self-evaluation rather than assessment is emphasised. The computer keeps track of their nominated answer and detailed feedback is provided on any question upon completion of the quiz. The style of the quiz forces students to carry out their own assessment and makes them responsible for their own learning. Again this was chosen to further promote a more student-centred learning environment and to encourage the independence valued in tertiary students.

This tutorial uses a number of different testing styles. For some questions corrections are given after one attempt, while for others the students cannot proceed until they enter the right answer. Some questions allow one or more wrong answers and then provide hints on how to do the problem. The students were also provided with a worksheet designed to provide them with a record of their progress.

The tutorial was developed using a standard process which involved researching content, writing text, designing animations, interface and navigation, collecting images and creating graphics, programming, debugging and proofing of the final version. Some of these stages overlapped, while others required a number of iterations before completion.

The project was developed using Macromedia Director 4.0, Adobe Photoshop 3.1 and Kaleidagraph 2.1 software on an Apple PowerMac 7100 with 16 Megabytes of RAM and a 500 Megabyte hard disk. QuickTime movies were deliberately avoided for this particular project because of time and financial constraints.

\section{Implementation}

After six lectures on the photoelectric effect and blackbody radiation, three one hour sessions were set aside for work on the tutorial in the university's undergraduate computer labs. The students accessed the software on one of two servers using Macintosh LCIII and Quadra 605 machines. Students were supervised during an initial explanatory session which also enabled 
the development team to observe how the software was used. Students were able to visit the laboratories out of class time and could access the server from anywhere on campus.

\section{Evaluation}

Evaluation of the software was carried out using several methods. Preand post-test questionnaires, using 7-point scales and open answers were developed under consultation with the Centre for Academic Development and Methods (CEDAM) at the Australian National University. Observations in class and informal discussions with the 35 participating students also elicited useful information about how the software was received. This stage of evaluation is purely formative and will be used to enhance the software and provide guidelines for future projects. Unfortunately, due to the small class size, there was no opportunity to use a control group, nor was it possible to use previous years results as a comparison due to changes in the course.

Pre-testing involved collecting background information on our sample group of students. This included previous knowledge of the material, previous experience with computers, their assessment of lectures and practical exercises and their expectations of multimedia. Post-testing asked students to rate the multimedia tutorial using the same format as assessment of the lectures. It also included opportunity to comment on various aspects of the tutorial in their own words.

A standard CEDAM questionnaire for the evaluation of large group teaching was also completed by students at the end of the course.

\section{Results}

\section{The pre-test survey}

The study group consisted of first and second year students of whom 15\% were female and whose average age was 20 years old. The response rate was $92 \%$.

All students were familiar with computers ( $94 \%$ using them at home and 97\% at university), $72 \%$ used the Internet and more than a third had previous experience with interactive multimedia. These figures confirmed our assumption that our students were not naive computer users and therefore brought with them many expectations about how computers could and should be used. Around half of the students had previously studied blackbody radiation or the photoelectric effect, either at high school or university.

The students were asked to evaluate the role of lectures and practicals in their learning. While lectures were rated very highly, comments showed the practicals to be confusing and difficult and indicated little use of 
textbooks to supplement learning. In this course, the majority of students took few notes in class, preferring to copy them from the server.

\section{The post-test survey}

Overall the student responses to the questionnaires were very encouraging. When rating the tutorial on the same scale as they did lectures and practicals all responses were more positive, especially those concerning the clarity and interest of the content. The interface and navigation were rated as easy to understand and use. The class time devoted to using the tutorial was deemed sufficient and attendance was very high for all sessions. Two thirds of the group used the tutorial out of class time with half of these accessing it two for more times.

Responses for which students used their own words were very useful for eliciting more detailed opinions. These indicated that animations and simulations proved very successful as they allowed students to use them in a variety of ways depending on their level of familiarity with the material. For some they illuminated difficult concepts, while for others they confirmed what they already understood. Most students found the feedback useful in the same way, with the detailed responses making clear the reasoning behind both right and wrong answers. One student, however, responded that the feedback "makes the questions useless, unless it is just to make us look at the material". The quiz was valued for increasing students' confidence in their understanding of the underlying concepts, although some complained it was too simple.

The associated worksheet also proved very popular, although many students $(46 \%)$ commented that if there had been no assessable task they probably would not use it. When asked if they would use the tutorial as a revision tool prior to an exam, responses were mixed with some students preferring this medium and other clearly stating their preference for texts. The vast majority found value in the diversions either for adding another dimension to their understanding or as a useful break from the monotony!

The most detailed comments and emphatic responses came when asked if multimedia tutorials should replace traditional teaching methods. All but one student wanted to retain lectures as an opportunity for human interaction and a forum for asking more detailed questions. A few commented that the combination used in the course allowed the lecture to achieve fuller potential than in other courses. The reaction to the replacement of labs by such a tool was mixed. Although students generally saw the value and achievement associated with practicals, they rated them poorly for enhancing understanding.

All students felt that multimedia, or this application at least, met or surpassed their expectations as a learning tool and felt it improved their understanding of the subject matter. 
Observations and informal discussion revealed that students felt a combination of multimedia and lectures was preferable to the traditional of lecture-tutorial-practical. Participation and motivation were increased from the previous year (MJB lectured ) and students asked more profound questions about concepts rather than simply seeking instructions on specific mathematical problems.

An independent, but less detailed, questionnaire of student attitude conducted by CEDAM also testified to the success of the new teaching strategies. The majority of those surveyed $(80 \%)$ rated the course as very good or outstanding with the remainder opting for good.

\section{Discussion}

A number of expectations held by the teaching team were challenged over the course of this project. Firstly, we were concerned that the material, originally designed with first year students in mind would be too simple for most second year students. It is clear, however, that the subject matter is still challenging even after exposure a number of times. We also expected that students would reject the prototype tutorial, because the graphics were unsophisticated. Instead they appreciated the clean interface, proving to us what can be achieved with a minimalist design approach and budget. Students also used it far more frequently than we expected and rejected it as a reference tool only.

The open answer part of the questionnaire revealed that although there are many different learning levels and styles within this group, the vast majority of students felt their own style was supported by the variety of approaches taken with this course.

Informal discussion revealed much about the insecurities of students. Many students are not comfortable with the idea of conducting their own evaluation. For example, the student who responded that tho feedback "makes the questions useless" had misunderstood the intention. A clear issue that emerged from the project is that students value face-to-face teaching highly, but want it to foster discussion, rather than transmission of information. It also became obvious that students will not use a tutorial if they feel it is a waste of time or it is just "tacked on". It is essential that educators look not only at the theoretical basis of learning, but the environment in which it supposed to occur. An understanding of student motivation and expectation must be accounted for when developing any new educational strategy.

A less surprising result of the evaluation of the course overall was the contrast between staff and student attitudes. The attitude of staff, gauged from informal discussions prior to implementing the new approach, was mixed. Students, on the other hand, are not only embracing educational technology, they are also expecting it more and more. Our concern is that multimedia techniques will indeed not succeed if this difference continues. 
A recent study has shown that educational technology must not only be fully integrated into a course, it must be incorporated into a departmental teaching strategy in order to be successful (Akerlind and Trevitt, 1995). A further advantage of this combination of educational technology and traditional methods comes from its inclusion of students with disabilities. Hearing-, visually- and manually impaired students can all benefit from this approach.

Our results indicate that students are in favour of the kind of self paced learning which can be achieved through interactive multimedia, while wanting to retain some aspects of traditional teaching. The model used in this instance has recognisable advantages for both students and staff. The lecture becomes a forum for discussing high level concepts with more opportunity for questions. This allows the lecturer to take on the role of facilitator and shifts the focus onto the student.

\section{Conclusions}

Changes in tertiary physics education in the past decade have left many departments short of the staff and resources to mount many of the classes previously considered fundamental to training in the field. Some of the most difficult and expensive practicals have been replaced by data analysis on paper or discarded completely. Tutorials have experienced a decline in attendance and students appear less motivated to extend themselves into non-assessable areas. Often this poor attendance has shifted the focus onto the lecture as the method for providing detailed information.

In response to these problems it was the aim of the authors in developing this software to provide a program rich in content and simulation to illuminate concepts covered in lectures, give insights into experimental method and reinforce professional practice in a way that would appeal to students and provide multiple opportunities for access.

It is clear from the results of our evaluation that interactive multimedia has the capacity to illuminate science accurately and effectively, offer a more attractive and stimulating learning environment and provide support for both student and teacher. It remains to be seen, however, whether this approach is reflected in better student performance.

\section{Acknowledgments}

The authors gratefully acknowledge the cooperation of the staff of the Department of Physics and Theoretical Physics and the students of PHYS2001/2015 of 1995 for their cooperation. We also thank David Baldwin and John Tucker of The Faculties Computer Unit for their assistance. SJB wishes to thank the Science Communication Group for their support and the Australian National University for the provision of a scholarship during the course of her Masters degree. 


\section{References}

Akerlind, G. and Trevitt, C. (1995). Enhancing Learning Through Technology: When Students Resist the Change. Proceedings of ASCILITE 1995, December 4-6.

Arundel, M., Fahndrich, C. and Day, D. (1994). An interactive, simulated experiment for biochemistry and Molecular Biology Students. Proceedings of APITITE 94, June 28-July 2.

Beattie, K. (1994). How to avoid inadequate evaluation of software for learning, In K. Beattie, C. McNaught and S. Wills (eds), Interactive multimedia in university education: Designing for change in teaching and learning. Elsevier Science BV, Amsterdam.

Bennett, S. J. (1995). The Use of Interactive Multimedia in the Communication of Science. Unpublished thesis, The Australian National University.

Clarke, C. and Swearingen, L (1994). Macromedia Director Design Guide. Hayden Books.

Halliday, D. and Resnick, R. (1988). Fundamentals of Physics. John Wiley and Sons, New York.

Laurillard, D. (1993). Rethinking University Teaching. Routledge, London.

Latchem, C., Williamson, J. and Henderson-Lancett, L. (1993). IMM: An overview, In C. Latchem, J. Williamson and L. Henderson-Lancett (eds), Interactive multimedia. Kogan Page, London.

Ladiges, P., Gleadow, R., Campbell, H., Dodds, A. and Lawrence, J. (1994). Innovative teaching methods in Biology including self study and multimedia programs. ANZAAS 63rd Congress, Geelong.

Stocklmayer, S. (1995). Private communication.

Whitnell, R., Fernandes, E., Almassizadeh, F., Love, J., Dugan, B., Sawrey, B. and Wilson, K. (1994). Multimedia Chemistry Lectures. Journal of Chemical Education, 71(9), 721.

Susan J. Bennett is with the Science Communication Group, Australian National University, Canberra ACT 0200, but is presently working with the Multimedia Interactive Learning Laboratory, ANU, Canberra ACT 0200. Dr Michael J. Brennan is with the Electron Physics Group, Research School of Physical Sciences E Engineering, Australian National University, Canberra ACT 0200, but is presently with the Land Space and Optoelectronics Division, DSTO, Salisbury SA 5108.

Please cite as: Bennett, S. J. and Brennan, M. J. (1996). Interactive multimedia learning in physics. Australian Journal of Educational Technology, 12(1), 8-17. http: / / www.ascilite.org.au/ajet/ajet12/bennett.html 